\title{
White starburst-like pattern as a dermoscopic clue in Old World cutaneous leishmaniasis *
}

\author{
Pablo Fernandez-Crehuet ${ }^{1,2}$
}

\author{
Ricardo Ruiz-Villaverde ${ }^{3}$
}

DOI: http://dx.doi.org/10.1590/abd1806-4841.20175728

\begin{abstract}
A 70-year-old woman was referred to our dermatological unit by her general practitioner last summer, with a history of a two-month ulcerated purplish lesion on her left arm that spread centrifugally and that unsuccessfully treated with topical corticosteroids. The dermoscopic evaluation revealed an erythematous macula with central ulceration that showed the characteristic called "white starburst-like pattern" and some vascular structures (dotted vessels, polymorphous/atypical vessels). The diagnosis of cutaneous leishmaniasis was made after histopathologic analysis and polymerase chain reaction essay. Keywords: Leishmania; Leishmania infantum; Leishmaniasis, cutaneous; Dermoscopy
\end{abstract}

A 70-year-old woman was referred to our dermatological unit by her general practitioner last summer, with a history of a twomonth ulcerated purplish lesion on her left arm that spread centrifugally and was unsuccessfully treated with topical corticosteroids. There was no history of previous trauma but she referred an insect bite after having a walk outdoors. The lesion caused intense itching but no other associated symptoms (sweating, fever or malaise) were reported. Clinical examination revealed a papular skin lesion with central serous crust with a peripheral erythematous halo on the internal surface of her left arm (Figure 1). The dermoscopic evaluation revealed an erythematous lesion with central ulceration that showed the characteristic presentation called "white starburst-like pattern" and some vascular structures (dotted vessels, polymorphous/atypical vessels; Figure 2). A skin biopsy was performed and Giemsa staining showed an inflammatory lymphocytic infiltrate in papillary and reticular dermis and macrophages filled with few amastigotes (Figure 3). Diagnosis of Old World Cutaneous Leishmaniasis was made. Pentavalent antimonials (meglumine antimoniate, 0.2-1 $\mathrm{mL}$ ) were injected under the edges of the lesion. After six months of follow-up, no recurrence was observed. Leishmaniasis is a protozoan parasitic disease transmitted by the bite of certain species of the infected female sand fly (subfamily Phlebotominae). The hosts are usually both domestic and wild mammals. Leishmaniasis is an endemic disease in 88 countries from all continents with an estimated incidence rate of 0.7-1.2 million cases per year. ${ }^{1}$ The widely clinical spectrum of cutaneous leishmaniasis sometimes makes its clinical diagnosis difficult. Classically, the geographic origin, parasite species and their interaction with the host's immune response yield two main forms of cutaneous leishmaniasis, namely Old World (Euro-

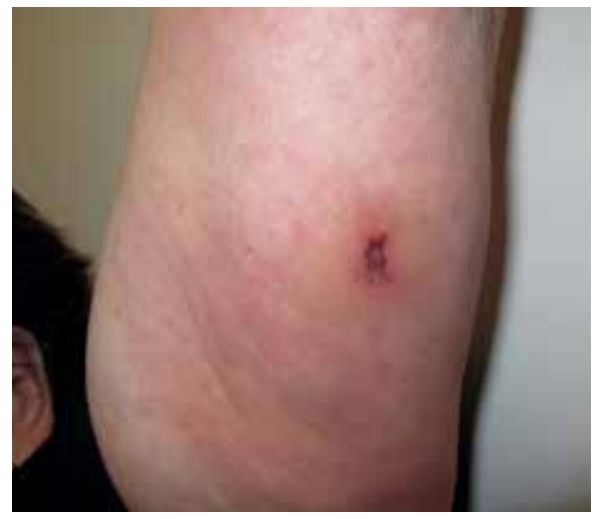

Figure 1: Purplish lesion with central erosion of $2 \times 1 \mathrm{~cm}$ located on the inner aspect of the left arm of the patient

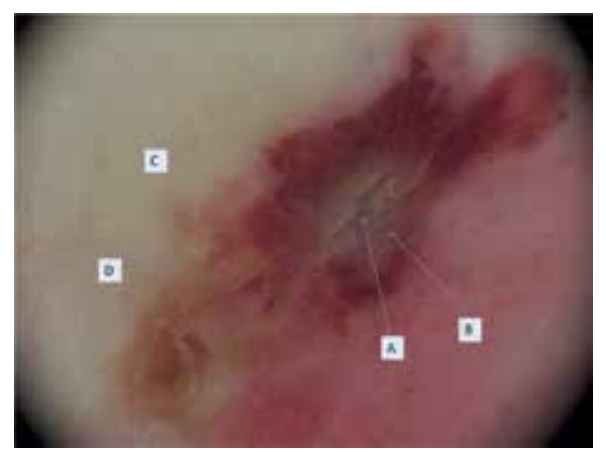

Figure 2: Dermoscopic image of the lesion (DermLite II ProHR, 3Gen LLC, California). A. Central erosion. B White starburst-like pattern. C. Dotted peripherical vessels. D. Atypical vessels

Received on 24.02.2016

Approved by the Advisory Board and accepted for publication on 13.06.2016

* Study conducted at the Hospital Universitario Reina Sofía. Instituto Maimónides de Investigación Biomédica (IMIBIC). Cordoba. Spain.

Financial support: none.

Conflict of interest: none.

Dermatology Department. Hospital Universitario Reina Sofía - Cordoba, Spain

Instituto Maimónides de Investigación Biomédica (IMIBIC) - Cordoba. Spain.

Dermatology Department. Hospital Virgen de las Nieves. Granada. Spain.

C2017 by Anais Brasileiros de Dermatologia 


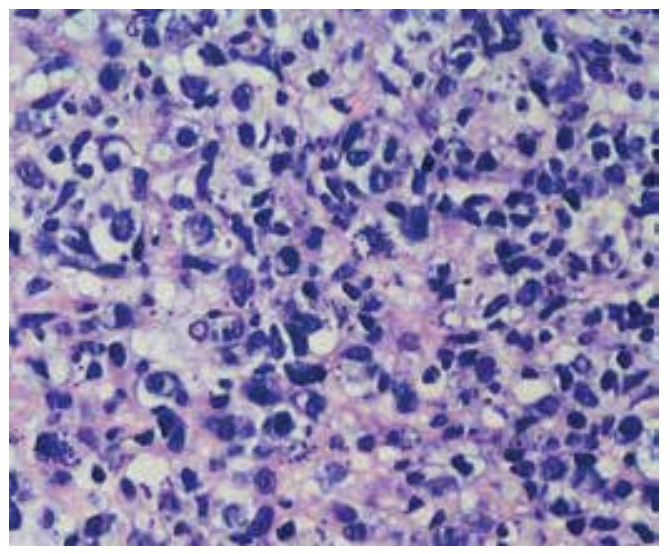

FigurE 3: Histopathology: Inflammatory lymphocytic infiltrate in papillary and reticular dermis where macrophages filled with few amastigotes can be appreciated. (Giemsa; original magnification: X40)

pean, Asian, and African) and New World (American) cutaneous leishmaniasis. Different species of leishmania parasites are responsible of the disease in these areas (i.e. Leishmania major in the Mediterranean African coast). Specifically, in the Spanish Mediterranean basin, virtually all cases of cutaneous leishmaniasis are due to Leishmania infantum, which is rarely the cause of leishmaniasis in other parts of the world. Spain is an endemic area for leishmaniasis, but reliable incidence or prevalence data are unknown as the disease is clearly underreported. ${ }^{2}$

Clinical differential diagnosis should be made with tinea incognito, because lesions often are masked with the use of topical corticosteroids, acute prurigo and tache noire as initial clinical feature of mediterranean spotted fever. Other diagnosis to rule out are sporotrichosis, blastomycosis, sarcoidosis, syphilis, pyogenic skin infections including ecthyma and idiopathic midline granuloma. ${ }^{3}$
The parasites can be observed in material obtained by scratches from the lesion margins. Giemsa and hematoxylin stains easily reveal non-flagellated (amastigote) forms. They appear as small dots within the macrophages and also extracellular. Polymerase chain reaction (PCR) is a very reliable method to demonstrate leishmania DNA in skin samples with a sensitivity rate of $95 \%$ and specificity of $100 \%$. ${ }^{4,5}$ In our patient, PCR detected Leishmania infantum. Skin biopsies should be taken from the margin of the lesion and may show different features according to the phase of the lesion. ${ }^{6}$ Acute lesions show epidermal hyperplasia, dermal inflammatory infiltrate consisting of lymphocytes, eosinophils and macrophages; these contain the Leishman-Donovan bodies (amastigotes) that stain blue with Giemsa. Histopathological features in chronic lesions depend on host immune status; in immunocompetent patients the predominant pattern is characterized by disorganized granulomas without necrosis and scarce parasites, but in immunocompromised patients macrophages with abundant intra and extracellular leishmanias are seen.

Dermoscopy is thus an extremely useful noninvasive technique in these cases. Generalized erythema and vascular structures are present in all cases reported. ${ }^{7}$ In initial lesions, yellow structures with an oval form, also known as "yellow tears", have been described and correspond to follicular plugs in histopathological study. In chronic or more advanced phases, similar to our case, "white starburst-like pattern" may be observed, corresponding to parakeratotic hyperkeratosis located around the erosion. Nevertheless, sensitivity and specificity rates of dermoscopy have not been established yet.

Intralesional infiltration of pentavalent antimonials with or without associated cryotherapy and the application of paromomycin sulfate $15 \%$ and methyl benzethonium chloride $12 \%$ twice a day for 20 days is an interesting therapeutic alternative with cure rates between $77-94 \% .{ }^{8} \square$

\section{REFERENCES}

1. García-Almagro D. Cutaneous leishmaniasis. Actas Dermosifiliogr. 2005;96:1-24.

2. Fernández-Crehuet $P$, Ruiz-Villaverde R. A 45 year old man with an ulcerated nodule on his right leg. BMJ. 2014 8;349:g5296.

3. Goto H, Lindoso JA. Current diagnosis and treatment of cutaneous and mucocutaneous leishmaniasis. Expert Rev Anti Infect Ther. 2010;8:419-33.

4. Foulet F, Botterel F, Buffet P, Morizot G, Rivollet D, Deniau M, et al. Detection and identification of Leishmania species from clinical specimens by using a realtime PCR assay and sequencing of the cytochrome B gene. J Clin Microbiol. 2007;45:2110-5

5. Hajjaran H, Vasigheh F, Mohebali M, Rezaei S, Mamishi S, Charedar S. Direct diagnosis of Leishmania species on serosity materials punctured from cutaneous leishmaniasis patients using PCR-RFLP. J Clin Lab Anal. 2011;25:20-4.

6. Faber WR, Oskam L, van Gool T, Kroon NC, Knegt-Junk KJ, Hofwegen H, et al. Value of diagnostic techniques for cutaneous leishmaniasis. J Am Acad Dermatol. 2003;49:70-4.
7. Llambrich A, Zaballos P, Terrasa F, Torne I, Puig S, Malvehy J. Dermoscopy of cutaneous leishmaniasis. Br J Dermatol. 2009;160:756-61.

8. Monge-Maillo B, López-Vélez R. Therapeutic options for old world cutaneous leishmaniasis and new world cutaneous and mucocutaneous leishmaniasis. Drugs. 2013;73:1889-920.
MAILING ADDRESS:
Pablo Fernandez-Crehuet
Av Menendez Pidal s/n
14004. Córdoba. Spain
Email:pablocrehuet@hotmail.com

How to cite this article: Fernandez-Crehuet P, Ruiz-Villaverde R. White starburst-like pattern as a dermoscopic clue in Old World Cutaneous Leishmaniasis. An Bras Dermatol. 2017;92(2):266-7. 